\title{
Strong dependence of spin dynamics on the orientation of an external magnetic field for InSb and InAs
}

\author{
K. L. Litvinenko, ${ }^{1, a)}$ M. A. Leontiadou, ${ }^{1}$ Juerong $L i,{ }^{1}$ S. K. Clowes, ${ }^{1}$ M. T. Emeny, ${ }^{2}$ \\ T. Ashley, ${ }^{2}$ C. R. Pidgeon, ${ }^{3}$ L. F. Cohen, ${ }^{4}$ and B. N. Murdin ${ }^{1}$ \\ ${ }^{1}$ Advanced Technology Institute, University of Surrey, Guildford GU2 7XH, United Kingdom \\ ${ }^{2}$ QinetiQ Ltd., St. Andrews Road, Malvern WR14 3PS, United Kingdom \\ ${ }^{3}$ Department of Physics, Heriot-Watt University, Edinburgh EH14 4AS, United Kingdom \\ ${ }^{4}$ Blackett Laboratory, Imperial College, London SW7 2BZ, United Kingdom
}

(Received 23 November 2009; accepted 30 January 2010; published online 16 March 2010)

\begin{abstract}
Electron spin relaxation times have been measured in InSb and InAs epilayers in a moderate $(<4 \mathrm{~T})$ external magnetic field. A strong and opposite field dependence of the spin lifetime was observed for longitudinal (Faraday) and transverse (Voigt) configuration. In the Faraday configuration the spin lifetime increases because the D'yakonov-Perel' dephasing process is suppressed. At the high field limit the Elliot-Yafet spin flip relaxation process dominates, enabling its direct determination. Conversely, as predicted theoretically for narrow band gap semiconductors, an additional efficient spin dephasing mechanism dominates in the Voigt configuration significantly decreasing the electron spin lifetime with increasing field. () 2010 American Institute of Physics. [doi:10.1063/1.3337111]
\end{abstract}

The key characteristics of any spintronic device are spin injection, manipulation, and detection. These characteristics are often studied in the presence of a significant external magnetic field but in spite of the fact that magnetic fields have a strong influence on spin dynamics ${ }^{1}$ there have been no systematic studies of these effects in semiconductors. In many cases the field dependence is ignored despite the fact that the results have been used to predict the spin dynamics of structures and devices in zero-field. We investigate here the magnetic field dependence of the spin lifetime in bulk III-V narrow gap semiconductors (NGSs) InSb and InAs, which act as model systems with strong spin effects because the heavy constituent atoms give rise to strong internal electric field and relativistic effects such as spin-orbit coupling. Other practical advantages of these materials for future device application are high electron effective g-value (in contrast to GaAs), ${ }^{2}$ small electron effective mass, and high mobility, so they are attractive for both high speed electronic ${ }^{3}$ and spintronic devices. ${ }^{4}$ We show that the dynamics are very sensitive to both the magnitude and direction of the magnetic field.

Spin dynamics can be measured in zero-field by timeresolved optical orientation using circularly polarized light. ${ }^{5-7}$ A perpendicular magnetic field is sometimes applied with the time-resolved technique in order to observe the Larmor precession ${ }^{2,8}$ (as in this work). In most other techniques, such as spin resonance experiments, where the line-width for microwave absorption is used to infer the spin relaxation time, ${ }^{9}$ a field is necessarily applied. In Hanle experiments a magnetic field (usually at $45^{\circ}$ to the sample plane), is used to produce components of spin out of plane when they are injected in-plane, and the results give insight into injection efficiency and dynamics. ${ }^{10}$ At the same time, a magnetic field is often important in spintronic device applications because it may be useful for switching magnetic contacts, as in the case of both spin-transistors ${ }^{3,11-13}$ and spin-LEDs. ${ }^{14}$ In any of

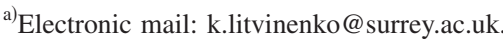

these situations the field dependence of the spin dynamics has a strong influence. It has already been reported that the fringe field from the patterned ferromagnetic contacts easily leads to deceptive results due to creation of a local Hall effect. ${ }^{15}$ We demonstrate that for NGSs in moderate magnetic field, simply changing the field direction can alter the electron spin lifetime by more than one order of magnitude.

The D'yakonov-Perel' (DP) process is a spin dephasing by internal magnetic fields which are nonzero in III-V semiconductors due to the lack of inversion symmetry. In the diffusive limit, each electron has a different momentum vector, $\mathbf{k}$ and hence experiences a different effective magnetic field, so there is a loss of ensemble spin polarization by inhomogeneous precession. Thus, in zero externally applied field the DP rate is ${ }^{16}$

$$
1 / \tau_{\mathrm{DP}}(0)=\left\langle\Omega^{2}(\mathbf{k})\right\rangle \tau_{p}^{*},
$$

where $\Omega$ is the precession vector due to the effective field and $\tau_{p}^{*}$ is the total momentum scattering time. The DP process dominates over the other spin relaxation processes in low mobility materials at elevated temperature. ${ }^{5,6,17}$

The DP process is suppressed in magnetic field for two main reasons. One is that the external field easily overcomes the very small effective fields, so that the Larmor precession of spins occurs homogeneously. The second is due to the cyclotron motion of the electrons, which causes the direction of $\mathbf{k}$ to change in a way equivalent to an increase in momentum scattering rate, that in turn suppresses the DP mechanism. ${ }^{18,19}$ For the condition $\Omega_{c}^{2} \tau_{p}^{*} \ll \Omega_{L}$ (i.e., B $\ll 25 \mathrm{~T}$ for InSb and $\mathrm{B} \ll 10 \mathrm{~T}$ for InAs),

$$
\frac{\tau_{\mathrm{DP}}(0)}{\tau_{\mathrm{DP}}(B)}=\frac{1}{8}\left[\frac{5}{1+\left(\Omega_{c} \tau_{a}^{*}\right)^{2}}+\frac{3}{1+\left(3 \Omega_{c} \tau_{a}^{*}\right)^{2}}\right],
$$

for both Faraday and Voigt configuration. Here $\tau_{\mathrm{DP}}(0)$ is the spin lifetime in zero externally applied magnetic field, $\Omega_{c}$ is the cyclotron frequency, $\Omega_{\mathrm{L}}$ is the Larmor frequency, and $\tau_{a}^{*}$ characterizes the relaxation of the antisymmetric part of the 


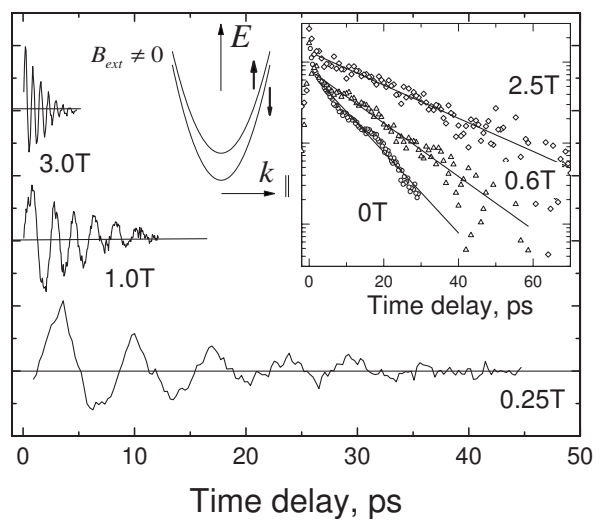

FIG. 1. The spin polarization measured as a function of time for InSb at 100 $\mathrm{K}$ at various magnetic field strengths in the Voigt configuration. Inset: As for the main figure for the Faraday configuration and $100 \mathrm{~K}$ (note log scale on the ordinate axis). The cartoon shows how the eigenstate energies for Landau subbands vary with momentum parallel to an externally applied magnetic field. The g-value has large magnitude at the bottom of the band (giving rise to relatively large Zeeman splitting) and reduces as $k_{\|}$increases.

distribution function and is proportional to the momentum relaxation time $\tau_{p}^{*}$. ${ }^{18}$ (In the case of scattering by impurities $\left.\tau_{a}^{*}=\tau_{p} / 3\right) .{ }^{20} \tau_{p}^{*}$ is in general equal to the faster of the electron-electron scattering time $\tau_{e e}$ and the mobility time $\tau_{p}$.

The Elliot-Yafet (EY) process $^{21,22}$ arises due to conduction-valence band mixing of the spin eigenstates, so that processes that would not normally allow a change in angular momentum can cause transitions between spin states. It has been assumed for GaAs that the EY process is not affected by the magnetic field at all up to $25 \mathrm{~T}^{23}$ and we follow this assumption in this work. We note that a detailed theoretical investigation ${ }^{1}$ has shown that there is a magnetic field dependence of the EY process in InSb in the extreme quantum limit but this work is far from that regime.

In the Voigt configuration an additional spin dephasing process is opened. This additional spin relaxation mechanism results from the momentum dependence of the electron g-factor, which arises from nonparabolicity (just as the effective mass increases away from the conduction band edge, the g-factor decreases in magnitude), shown as an inset in Fig. 1. Because of the variation in the g-factor, spins polarized perpendicular to the applied field with different $k_{\|}$, the component of momentum along the field direction, precess about the external field at different rate and thus lose their coherence. In this sense the process is very similar to DP though it arises from nonparabolicity (quartic terms in the dispersion) while the DP process arises from inversion asymmetry (the cubic terms, which have much smaller coefficients). Therefore this dephasing mechanism is predicted to be more effective in NGSs. ${ }^{23}$ This process was introduced by Margulis and Margulis ${ }^{20,24}$ and will be referred to as the MM process. The MM process, unlike the EY mechanism, does not involve a spin flip in the electron scattering, and, unlike the DP process, exists only in the presence of a magnetic field. The MM process rate is, by analogy with the DP rate Eq. (1),

$$
\frac{1}{\tau_{\mathrm{MM}}}=\left(\left\langle\Omega_{L}^{2}\left(k_{\|}\right)\right\rangle-\left\langle\Omega_{L}\left(k_{\|}\right)\right\rangle^{2}\right) \tau_{p}^{*} .
$$

Here $\Omega_{L}$ is the Larmor precession rate. $\left[\mathrm{NB}\left\langle\Omega_{L}\left(k_{\|}\right)\right\rangle \neq 0\right.$, while the equivalent term in the DP rate Eq. (1) is zero]. Assuming that the density of states is approximately of the form $\rho \propto 1 / E^{1 / 2}$ (the zero energy was set at the "bottom" of
TABLE I. Coefficients for the MM process. The MM rate is $1 / \tau_{\mathrm{MM}} \propto g_{1}^{2} B^{2}$.

\begin{tabular}{lccc}
\hline \hline & $\begin{array}{c}\text { InSb } \\
(\mathrm{me} 1655)\end{array}$ & $\begin{array}{c}\text { InAs } \\
(\mathrm{IC} 313)\end{array}$ & GaAs \\
\hline$d \tau_{\mathrm{MM}} / d\left(B^{-2}\right)\left(\mathrm{psT}^{2}\right)$ [Expt at $\left.100 \mathrm{~K}\right]$ & 3.7 & 41 & $\ldots$ \\
$g_{1}\left(\mathrm{eV}^{-1}\right)$ & $260^{\mathrm{a}}$ & $80^{\mathrm{b}}$ & $6.3^{\mathrm{c}}$ \\
$1 / g_{1}^{2}($ normalized to InSb) & 1 & 10 & 1700 \\
\hline \hline
\end{tabular}

${ }^{\mathrm{a}}$ Reference 2.

${ }^{\mathrm{b}}$ Reference 26

${ }^{\mathrm{c}}$ Reference 27.

the Landau band) and the energy dependence of the gyromagnetic ratio is $g^{*}(E)=g_{0}+g_{1} E$, then we find

$$
\frac{1}{\tau_{\mathrm{MM}}}=\frac{\left(2 g_{1} E_{F} \mu_{B} B\right)^{2}}{45 \hbar^{2}} \tau_{p}^{*} .
$$

Clearly this formula contains significant simplification but it can be seen that $1 / \tau_{\mathrm{MM}} \propto g_{1}^{2} B^{2}$. For the materials of interest here $g_{0}$ is negative and $g_{1}$ is positive (see Table I). Accurate numerical calculations show that at high magnetic fields the efficiency of the MM process saturates. ${ }^{20,23}$

A mid-infrared circularly polarized pump-probe transient absorption technique was used to study the spin dynamics in NGSs with laser wavelength in the range 3-7 $\mu \mathrm{m}$. The samples used in this study were an undoped InSb $5 \mu \mathrm{m}$ thick epilayer (me1655) and a Si-doped InAs $4 \mu \mathrm{m}$ thick epilayer (IC311), both grown by molecular-beam epitaxy on a semi-insulating GaAs substrate. A detailed description of the samples and our experimental technique can be found elsewhere. ${ }^{2,5,6}$ In order to investigate the influence of the external magnetic field each sample was placed in a split coil superconducting magnet with optical access in either the Faraday $(\mathbf{S} \| \mathbf{B})$ or Voigt $(\mathbf{S} \perp \mathbf{B})$ configurations. The temperature of the sample was controlled in the range from $10 \mathrm{~K}$ up to room temperature. The induced transmission changes of the probe beam as a function of the time delay between pump and probe beam are shown in Fig. 1 for both Voigt and Faraday configuration. In the Faraday geometry a simple exponential decay of the polarization is observed, while in the Voigt configuration the polarization oscillates with the Larmor frequency as it decays. ${ }^{2}$

The magnetic field dependence of the electron spin lifetime derived from the data of Fig. 1 is summarized in Fig. 2. The spin lifetime was taken from the least-squares fit of exponential decays (Faraday) or exponentially decaying sinusoids (Voigt) from the data of Fig. 1. The error bars on Fig. 2 (10\%-5\%) were taken from this fitting, and correspond well with the standard deviation in cases where multiple decays were taken under the same conditions.

The experimental zero field spin lifetime agrees well with previously reported values. ${ }^{6,7}$ The DP process, which is the dominant spin relaxation mechanism at zero magnetic fields, is quickly suppressed with external magnetic field in either configuration. In the Faraday configuration [see Fig. 2(a)], the MM process is inefficient ${ }^{23}$ and essentially the spin lifetime saturates at a value given by the EY process.

By contrast, in the Voigt configuration [see Fig. 2(b)], the MM process makes a significant contribution to spin relaxation. It begins to dominate even at low magnetic field. In GaAs the interplay between the DP and the MM process is predicted to result in a maximum of spin lifetime at around $21 \mathrm{~T}$ at $0 \mathrm{~K}$ and $10 \mathrm{~T}$ at $100 \mathrm{~K}$, and the very limited experi- 

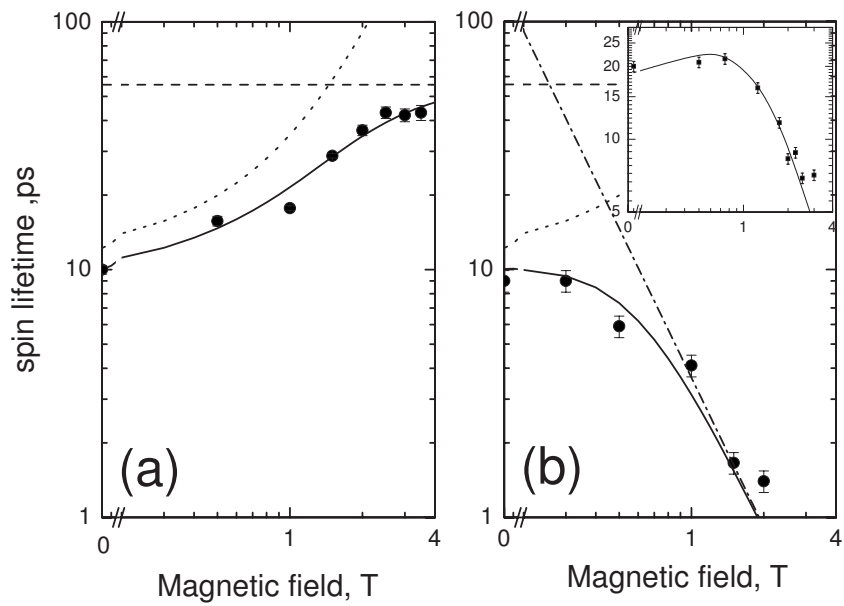

FIG. 2. The spin lifetime taken from fitting exponential decays (or exponentially decaying sinusoids) to the data of Fig. 1, plotted as a function of external magnetic field. (a) Faraday, and (b) Voigt configuration in InSb at $100 \mathrm{~K}$. Theoretical dependences of the different spin relaxation mechanisms at $100 \mathrm{~K}$ are also shown: EY (dashed line), DP (dotted line), MM (dotteddashed line), and total spin relaxation time (solid line). Inset: The spin lifetime as a function of external magnetic field in Voigt configuration in InAs at $100 \mathrm{~K}$.

mental data in GaAs (Ref. 8) compared to the theory shows the correct trend. ${ }^{23}$ The maximum, however, has not previously been observed experimentally. The efficiency of the MM process is much larger in NGSs due to considerably stronger nonparabolicity and consequently in InSb the maximum of spin lifetime is already achieved at $0.2 \mathrm{~T}$ at $100 \mathrm{~K}$. The same maximum in the InAs epilayer is found to be at $1 \mathrm{~T}$ at $100 \mathrm{~K}$ [see the inset of Fig. 2(b)] and proper exploration of these regimes can be made and compared to theory.

We produce a fit to the InSb and InAs data as shown by the lines in Fig. 2. The procedure was to fit the Faraday geometry data with the DP [Eq. (2)] and EY (assumed to be a constant independent of field-see above) processes only $\left(1 / \tau_{\text {Faraday }}=1 / \tau_{\mathrm{DP}}+1 / \tau_{\mathrm{EY}}\right)$, and then to fit the Voigt geometry data by adding in the MM process $\left(1 / \tau_{\text {Voigt }}=1 / \tau_{\text {DP }}\right.$ $\left.+1 / \tau_{\mathrm{EY}}+1 / \tau_{\mathrm{MM}}\right)$ without changing the DP and EY curves. A good global fit to the data is thus obtained (Fig. 2) with only four fitting parameters $\left[\tau_{p}^{*}, \tau_{\mathrm{DP}}(0), \tau_{\mathrm{EY}}\right.$, and the MM coefficient]. We have previously measured the mobility momentum relaxation time $\tau_{p}$ which is related to the mobility $\mu$ by $\tau_{p}=\mu m_{e} / e$ and found that $\tau_{p}=0.24$ ps for InSb (me1655) (Ref. 2) and $\tau_{p}=0.3$ ps for InAs (IC311). ${ }^{6}$ These values are (within experimental error) the same as the fitted times for $\tau_{p}^{*}$ in the Faraday configuration implying that electron-electron scattering is negligible for our samples. The mobility is assumed to be field independent for our magnetic field range. The coefficients for the MM fits are given in Table I, and are compared with the predicted scaling with $g_{1}^{2}$. Clearly the MM process is much more effective in NGSs than in GaAs, and, as expected, in InSb it is an order of magnitude faster than in InAs. The other parameters from the fit are the zero field DP spin lifetime $(12.1 \pm 1.5)$ ps and EY spin lifetime $(56 \pm 4)$ ps for the InSb sample [the latter agrees well with prediction of 70 ps (Ref. 25)] and zero-field DP spin lifetime $(18.5 \pm 1.2)$ ps for the InAs sample. We note that the EY lifetime fit is quite sensitive to uncertainty in $\tau_{a}^{*}$. In contrast, the MM process coefficient (the main interest of this work) is not affected in this way because it dominates over a wide range of field in the Voigt geometry.
In conclusion, we have explored the extreme sensitivity of the electron spin lifetime in InSb and InAs to the magnetic field orientation. In the Faraday configuration the spin lifetime saturates at the high field limit at a value determined by the EY process. This allowed us to directly measure the EY lifetime at elevated temperature where DP normally dominates. Knowledge of the EY lifetime is important because it sets the limit for suppression of the DP process in quantum wells employing structural modification of spin-orbit interaction such as by use of [110] growth. Typical DP lifetimes in InSb quantum wells are around $1 \mathrm{ps},{ }^{5}$ so we conclude that suppression of the spin relaxation rate by a factor of $\sim 50$ should be possible. In the Voigt configuration the MM process dominates and shortens the spin lifetime considerably with $\mathrm{B}^{-2}$. The electron spin lifetime in InSb epilayers can be modified by more than one order of magnitude simply by changing the direction of a moderate, externally applied magnetic field. This knowledge is crucial for correct interpretation of spin experiments and for successful design of future spintronic devices.

${ }^{1}$ P. Boguslawski and W. Zawadzki, J. Phys. C 13, 3933 (1980).

${ }^{2}$ K. L. Litvinenko, L. Nikzad, C. R. Pidgeon, J. Allam, L. F. Cohen, T. Ashley, M. Emeny, W. Zawadzki, and B. N. Murdin, Phys. Rev. B 77, 033204 (2008).

${ }^{3}$ J. M. S. Orr, P. D. Buckle, M. Fearn, C. J. Storey, L. Buckle, and T. Ashley, New J. Phys. 9, 261 (2007).

${ }^{4}$ H. C. Koo, J. H. Kwon, J. Eom, J. Chang, S. H. Han, and M. Johnson, Science 325, 1515 (2009).

${ }^{5}$ K. L. Litvinenko, B. N. Murdin, J. Allam, C. R. Pidgeon, M. Bird, K. Morris, W. Branford, S. K. Clowes, L. F. Cohen, T. Ashley, and L. Buckle, New J. Phys. 8, 49 (2006).

${ }^{6}$ B. N. Murdin, K. Litvinenko, J. Allam, C. R. Pidgeon, M. Bird, K. Morrison, T. Zhang, S. K. Clowes, W. R. Branford, J. Harris, and L. F. Cohen, Phys. Rev. B 72, 085346 (2005).

${ }^{7}$ B. N. Murdin, K. Litvinenko, D. G. Clarke, C. R. Pidgeon, P. Murzyn, P. J. Phillips, D. Carder, B. Redlich, A. F. G. van der Meer, S. Clowes, J. Harris, L. F. Cohen, T. Ashley, and L. Buckle, Phys. Rev. Lett. 96, 096603 (2006).

${ }^{8}$ J. M. Kikkawa and D. D. Awschalom, Phys. Rev. Lett. 80, 4313 (1998).

${ }^{9}$ P. Schneider, J. Kainz, S. D. Ganichev, S. N. Danilov, U. Rossler, W. Wegscheider, D. Weiss, W. Prettl, V. V. Belkov, M. M. Glazov, L. E. Golub, and D. Schuh, J. Appl. Phys. 96, 420 (2004).

${ }^{10}$ O. Maksimov, H. Lu, M. Munoz, M. C. Tamargo, and N. Samarth, J. Supercond. Novel Magn. 18, 195 (2005).

${ }^{11}$ M. Ohno and K. Yoh, Phys. Rev. B 77, 045323 (2008).

${ }^{12}$ J. Wan, M. Cahay, and S. Bandyopadhyay, Physica E (Amsterdam) 40 2659 (2008)

${ }^{13}$ R. R. Pela and L. K. Teles, J. Magn. Magn. Mater. 321, 984 (2009).

${ }^{14}$ A. V. Stier, C. J. Meining, B. D. McCombe, I. Chado, P. Grabs, G. Schmidt, and L. W. Molenkamp, Appl. Phys. Lett. 93, 081112 (2008).

${ }^{15}$ F. G. Monzon and M. L. Roukes, J. Magn. Magn. Mater. 195, 19 (1999).

${ }^{16}$ M. I. D'yakonov and V. I. Perel', Sov. Phys. JETP 33, 1053 (1971).

${ }^{17}$ T. F. Boggess, J. T. Olesberg, C. Yu, M. E. Flatte, and W. H. Lau, Appl. Phys. Lett. 77, 1333 (2000).

${ }^{18}$ E. L. Ivchenko, Sov. Phys. Solid State 15, 1048 (1973).

${ }^{19}$ V. A. Maruschak, M. N. Stepanova, and A. N. Titkov, Sov. Phys. JETP 37, 400 (1983).

${ }^{20}$ A. D. Margulis and V. A. Margulis, Sov. Phys. Solid State 25, 918 (1983).

${ }^{21}$ R. J. Elliott, Phys. Rev. 96, 266 (1954).

${ }^{22}$ Y. Yafet, Solid State Phys. 14, 1 (1963).

${ }^{23}$ F. X. Bronold, I. Martin, A. Saxena, and D. L. Smith, Phys. Rev. B 66 233206 (2002).

${ }^{24}$ M. W. Wu and C. Z. Ning, Eur. Phys. J. B 18, 373 (2000).

${ }^{25}$ P. H. Song and K. W. Kim, Phys. Rev. B 66, 035207 (2002).

${ }^{26}$ C. R. Pidgeon, D. L. Mitchell, and R. N. Brown, Phys. Rev. 154, 737 (1967).

${ }^{27}$ M. J. Yang, R. J. Wagner, B. V. Shanabrook, J. R. Waterman, and W. J. Moore, Phys. Rev. B 47, 6807 (1993). 